\title{
Prominence Oscillations activated by an EUV wave
}

\section{Pooja Devi ${ }^{\mathrm{a}, *}$, Ramesh Chandra ${ }^{\mathrm{a}}$, Reetika Joshi ${ }^{\mathrm{a}}$, P. F. Chen ${ }^{\mathrm{b}}$, Brigitte Schmieder ${ }^{\mathrm{c}, \mathrm{d}}$, Wahab Uddin ${ }^{\mathrm{e}}$, Yong-Jae Moon ${ }^{\mathrm{f}}$}

\author{
${ }^{a}$ Department of Physics, DSB Campus, Kumaun University, Nainital-263001, India \\ ${ }^{b}$ School of Astronomy $\mathcal{G}$ Space Science, Nanjing University, Nanjing 210023, China \\ ${ }^{c}$ Observatoire de Paris, LESIA, UMR8109 (CNRS), F-92195 Meudon Principal Cedex, France \\ ${ }^{d}$ Centre for mathematical Plasma Astrophysics, Dept. of Mathematics, KU Leuven, 3001 Leuven, Belgium \\ ${ }^{e}$ Aryabhatta Research Institute of Observational Sciences (ARIES), Nainital 263 001, India \\ ${ }^{f}$ School of Space Research, Kyung Hee University, Yongin, Gyeonggi-Do, 446-701, Korea
}

\begin{abstract}
Prominence oscillations are one of interesting phenomena in the solar atmosphere, which can be utilized to infer the embedded magnetic field magnitude. We present here the transverse oscillations of two different prominences located at the East solar limb on 2011 February 11 using the multi-wavebands data of the Atmospheric Imaging Assembly (AIA) on-board the Solar Dynamics Observatory (SDO) satellite. A prominence eruption was observed towards the east direction with an average speed of $\approx 275 \mathrm{~km} \mathrm{~s}^{-1}$. The eruption is fitted with the combination of a linear and an exponential functions of time. An extreme ultraviolet (EUV) wave event was associated with the prominence eruption. This EUV wave triggered the oscillations of both prominences on the East limb. We computed the period of each prominence using the wavelet analysis method. The oscillation period varies from 14 to $22 \mathrm{~min}$. The magnetic field of the prominences was derived, which ranges from 14 to $20 \mathrm{G}$.
\end{abstract}

(C) 2022 COSPAR. Published by Elsevier Ltd All rights reserved.

Keywords: Prominence eruption; EUV wave; Oscillations

\section{Introduction}

Solar prominences, also known as filaments on the solar disk, are high density cool materials hanging in the solar corona (for reviews see: Labrosse et al., 2010; Chen et al., 2020). They are observed in various chromospheric and coronal filters in quiet regions as well in active regions. They can erupt partially or fully, whenever the balance between magnetic tension and magnetic pressure gradient is disturbed. The eruption of prominences, either full or partial, is often accompanied by solar flares from medium to large classes (for example, Aulanier et al., 2000; Liu et al., 2009; Schmieder et al., 2015; Joshi et al., 2017; Roudier et al., 2018). When an eruption fails, sometimes the filament may return to a quasi-static equilibrium later on (for example, Joshi et al., 2013).

\footnotetext{
${ }^{*}$ Corresponding author:

Email address: setiapooja.ps@gmail .com (Pooja Devi)
}

An interesting phenomenon associated with prominence eruption is their association with extreme ultraviolet (EUV) waves (Zheng et al., 2012; Chandra et al., 2016; Zheng et al., 2020). The EUV waves are defined as the propagating bright fronts, first discovered by the Extreme ultraviolet Imaging Telescope (EIT) on board the Solar and Heliospheric Observatory (SOHO) satellite. Initially, they were explained as fast-mode magnetohydrodynamic (MHD) waves (Thompson et al., 1998; Wang, 2000; Wu et al., 2001; Ofman \& Thompson, 2002; Vršnak et al., 2002; Srivastava et al., 2016, and references cited therein). However, the finding of stationary fronts and the extremely slow speeds of some EUV waves could not be explained by the fast-mode MHD wave model (Delannée \& Aulanier, 1999; Delannée, 2000). Therefore, to reconcile these discrepancies, Chen et al. (2002) and Chen et al. (2005) proposed a hybrid model. According to their model, EUV waves have two components, a non-wave (known as EIT wave) and a fast-mode MHD wave. Sometimes, the fast mode EUV wave 
can be converted into a slow-mode wave when it interacts with a magnetic separatrix (Chen et al., 2016). Later SDO observations confirmed this model in many events (Zhukov \& Auchère, 2004; Chen \& Wu, 2011; Zhao et al., 2011; Chandra et al., 2021a).

EUV waves phenomena can trigger oscillations of nearby or distant prominences/filaments, which have been observed for a long time (Thompson \& Schmieder, 1991; Zhang \& Engvold, 1991; Vršnak, 1993; Shen et al., 2014b). Prominence oscillations provide a useful tool to diagnose the coronal magnetic field (see the review by Arregui et al., 2018). These oscillations are categorized into two types, namely, longitudinal and transverse. In longitudinal oscillations, the direction of the oscillations is parallel to the prominence threads whereas in transverse oscillations, it is normal to the prominence threads (Zhou et al., 2018), not to the prominence spine as claimed by many papers. Longitudinal oscillations are often excited by nearby impulsive events, for example, microflares and small jets (Luna \& Karpen, 2012; Liakh et al., 2020; Zhang et al., 2020; Luna \& Moreno-Insertis, 2021), whereas transverse oscillations are often triggered by energetic disturbances produced by distant flares, EUV waves and CMEs (Okamoto et al., 2004; Liu et al., 2012a), providing a useful tool to diagnose the coronal magnetic field. According to previous studies, longitudinal oscillations usually have a longer period in comparison to transverse oscillations (Luna et al., 2014). It is also reported that longitudinal and transverse oscillations can be observed simultaneously in separate filaments or in a single filament (Shen et al., 2014b; Pant et al., 2016; Zhang et al., 2017a,b; Mazumder et al., 2020; Zhang et al., 2020).

The restoring forces acting for these two kinds of oscillations are quite different. In the longitudinal oscillations, gravity is the main restoring force (Luna \& Karpen, 2012; Zhang et al., 2012). Using 1-dimensional hydrodynamic numerical simulations, Luna \& Karpen (2012) and Zhang et al. (2012) demonstrated that the field-aligned component of gravity can explain the obtained period, favoring the pendulum model. Later on, Luna (2016) did 2-dimensional (2D) MHD simulations and concluded that it is similar to the simplified pendulum model. 2D non-adiabatic MHD simulations were also performed by Zhang et al. (2019) to explain the longitudinal oscillations. Theoretical models for transverse oscillations in filaments have been provided by Hyder (1966) and Kleczek \& Kuperus (1969). In these models, the magnetic tension is considered as the main restoring force.

In this article, we present the transverse oscillations of the prominences situated at the east limb. These oscillations were triggered by an EUV wave associated with a filament eruption on 2011 February 11. The article is organized as follows: Section 2 presents the observational data sets, event overview, the prominence oscillations and coronal seismology. In Section 3, the discussion and summary of the study are given.

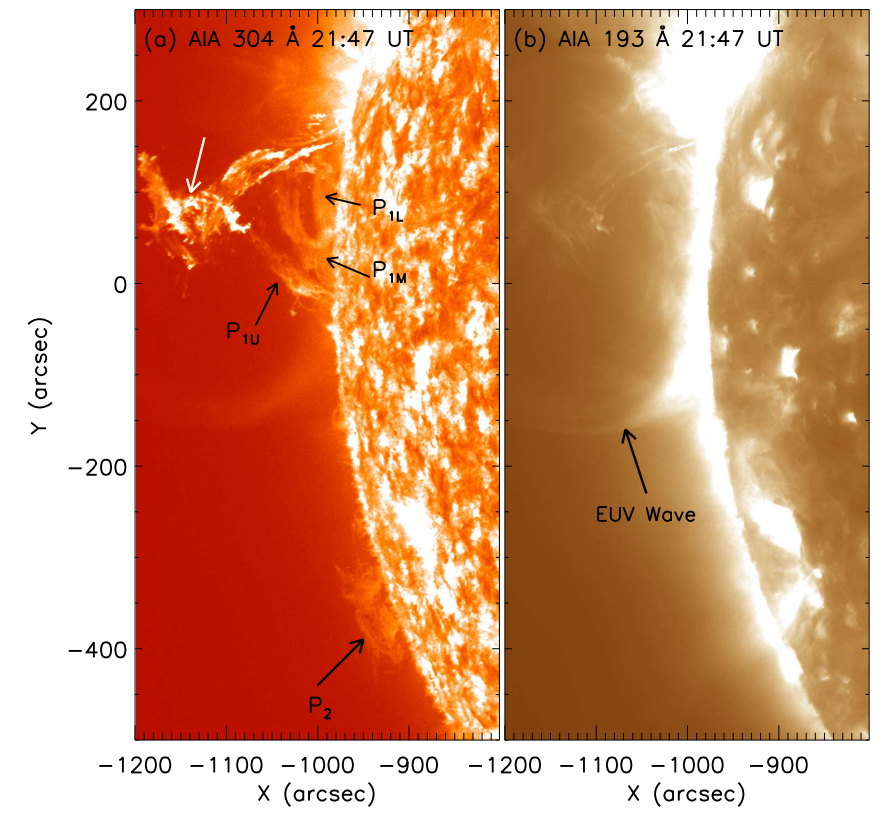

Fig. 1. Images of the prominences and EUV waves at AIA 304 and $193 \AA$ in panels (a) and (b), respectively. Three prominence layers $\mathrm{P}_{1 L}, \mathrm{P}_{1 M}, \mathrm{P}_{1 U}$, and another prominence $\mathrm{P}_{2}$ are shown by the black arrows in panel (a). A white arrow in panel (a) presents the erupting prominence. The EUV wave is shown in panel (b).

\section{Observations}

\subsection{Data sets}

The oscillations were observed in most of the filtergrams of the Atmospheric Imaging Assembly (AIA, Lemen et al., 2012) on board the Solar Dynamics Observatory (SDO, Pesnell et al., 2012). Considering the visibility, in the current study, we used the data in AIA $304 \AA$ and $193 \AA$. The spatial resolution and the cadence of the AIA data are $1.2^{\prime \prime}$ and $12 \mathrm{sec}$, respectively. The data are processed using different routines available in the solar software (SSW).

\subsection{Event Overview}

On 2011 February 11, at the east limb a prominence eruption was observed and the eruption was associated with a GOES B8.0-class flare. The prominence eruption was initiated at $\approx$ 21:40 UT in the east direction and later on it evolved to a slow coronal mass ejection (CME) at 22:12 UT in the Large Angle and Spectrometric Coronagraph (LASCO) C2 field-ofview with a linear average speed of $\approx 469 \mathrm{~km} \mathrm{~s}^{-1}$. In Figure 1 , the erupting and the oscillating prominences are shown in the left panel with white and black arrows, respectively, where $\mathrm{P}_{1 L}$, $\mathrm{P}_{1 M}$, and $\mathrm{P}_{1 U}$ stand for the lower, middle, and upper layers of the first prominence, respectively. $\mathrm{P}_{2}$ is the second oscillating prominence located in a different active region. The EUV wave is highlighted in the right panel.

The evolution of the prominence eruption in AIA $304 \AA$ is presented in the upper panel of Figure 2. For the kinematics of the eruption, we selected an artificial slice in the eruption direction. The slice is drawn in panel (c) of the same figure and 

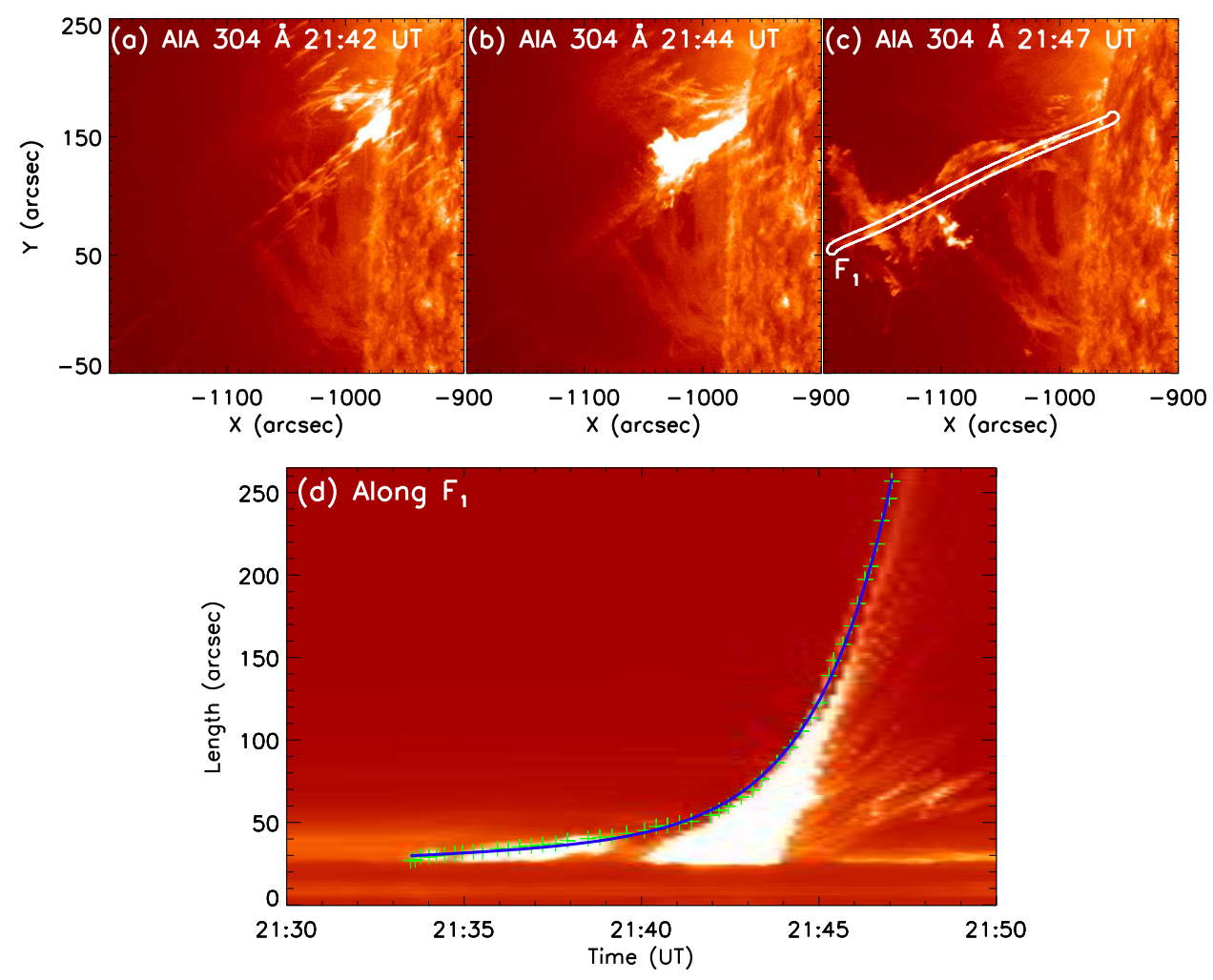

Fig. 2. Images showing the sequence of the prominence eruption in panels (a)-(c). The slice $F_{1}$ in panel (c) is chosen along the direction of prominence eruption, and panel (d) displays the time-distance diagram of the AIA $304 \AA$ intensity along the slice $\mathrm{F}_{1}$. We fit a function " $a e^{b\left(t-t_{0}\right)}+c t+h_{0}$ " in the leading edge of the eruption. These data points selected for the fitting are shown by green "plus" symbols whereas the blue solid line is the fitting of the function in the selected data points.

named as $F_{1}$. The time-distance plot along this slice is displayed in panel (d) of the figure. The combination of a linear and an exponential function is used to fit the height evolution of the erupting prominence. The function is in the form of $a e^{b\left(t-t_{0}\right)}+$ $c t+h_{0}$, where $a, b, c$, and $h_{0}$ are constant parameters to be found by the fitting. This function is similar to the function used by Cheng et al. (2020) and Chandra et al. (2021b). We fix $t_{0}$ to our first data point which is at 21:33:24 UT. The data points for the fitting are selected manually at the outer edge of the brightening around the slice. These data points are shown by green "plus" symbols and the blue solid line is the fitting of the function in the selected data points. The parameters $a, b$, and $c$ are determined in the fitting to be $64.46 \mathrm{~km}, 0.46 \mathrm{~min}^{-1}$ , and $10.36 \mathrm{~km} \mathrm{~s}^{-1}$, respectively. The value of the reduced $\chi$ square for the current fitting is 1.6. The average speed of the eruption is $\approx 275 \mathrm{~km} \mathrm{~s}^{-1}$.

Apart from the erupting prominence, at the east limb we find another two prominences. One is close to the erupting prominence and contains three layers one above another (like decker configuration) and they are labelled as $\mathrm{P}_{1 L}, \mathrm{P}_{1 M}$, and $\mathrm{P}_{1 U}$, respectively. The second prominence was in the southern hemisphere and is labelled as $\mathrm{P}_{2}$. The locations of these prominences in AIA $304 \AA$ along with the erupting prominence are depicted in Figure 1 (left panel).

The eruption of the main prominence drove an EUV wave, which is shown in AIA $193 \AA$ in the right panel of Figure 1.
The details of this EUV wave event were studied by Chandra et al. (2021a) using the multi-wavelength and multi-view point observations. As discussed in Chandra et al. (2021a), such an EUV wave event has two components i.e. a slower non-wave which is interpreted to be due to magnetic field line stretching and a fast-mode MHD wave. The speed of the slower wave is $298 \pm 5 \mathrm{~km} \mathrm{~s}^{-1}$, whereas the fast-mode wave speed is $445 \pm 6 \mathrm{~km}$ $\mathrm{s}^{-1}$.

\subsection{Prominence Oscillations and Coronal Seismology}

When the fast component of the EUV wave, i.e. the fastmode MHD wave, moved towards the east-south direction, it encounters the prominences situated on the east limb. As a result, the prominences $\mathrm{P}_{1 L}, \mathrm{P}_{1 M}, \mathrm{P}_{1 U}$, and $\mathrm{P}_{2}$ oscillated. The oscillations are clearly visible in the attached movie. In order to study the prominence oscillations in detail, we select two slices shown in panels (a) and (c) of Figure 3. The time-distance plots along these slices are drawn in panels (b) and (d) of the figure, respectively. In panel (b), we can see the oscillations of the $\mathrm{P}_{1 L}, \mathrm{P}_{1 M}$, and $\mathrm{P}_{1 U}$ prominence layers. In the same way the oscillation of prominence $P_{2}$ is depicted in panel (d) of the same figure. We track manually the evolution of the center of each oscillating prominence, which is over-plotted as the black-dashed lines in the same figure.

The above manually tracked points are used for the timeperiod analysis of these oscillations. To calculate the time pe- 
riod, we apply the wavelet analysis technique, with a significance test for the levels higher than or equal to $95 \%$ as real (Figure 4). The details about the wavelet technique and significance test were well explained in Torrence \& Compo (1998). The significant region is bounded by the cone-of-influence (COI) region, which acts as an important background for the edge effect for the given time range (Luna et al., 2017; Joshi et al., 2020). This method is used to find the period of all the four oscillations in $\mathrm{P}_{1 U}, \mathrm{P}_{1 M}, \mathrm{P}_{1 L}$, and $\mathrm{P}_{2}$. The derived periods of the oscillations vary from 14 to $22 \mathrm{~min}$ and are provided in Table 1. An example of the wavelet analysis is given in Figure 4. In this figure, we display the time evolution of the displacement and its wavelet spectrum for the oscillations of prominence $\mathrm{P}_{1 M}$, where the black dashed line marks the peak period at $14.0 \mathrm{~min}$. As for the amplitude of the oscillations in the two selected slices $S_{1}$ and $S_{2}$, we found that the amplitude is significantly weaker along slice $S_{2}$. The reason is straightforward, i.e., the slice $S_{2}$ is much further away from the eruption source region. As the EUV wave propagates out, it becomes weaker and weaker, hence the oscillation amplitude along slice $S_{2}$ is significantly smaller.

It is believed that the transverse oscillations are due to the dominant magnetic tension restoring force. Taking this fact into account, we consider the reported oscillations here like the kink-mode oscillation as suggested by Zhou et al. (2016) and Zhang \& Ji (2018). Coronal seismology allows us to determine the physical properties of the prominences such as the magnetic field strength (Nakariakov \& Verwichte, 2005; Nakariakov et al., 2021). We find the lengths of prominences by assuming that the prominences are along the magnetic field lines and the length of the coronal loops are approximately equal to prominence length. Then we could estimate the length of each prominence viewed as loops at the limb, one over the other. We find that the length of the lower loop $\mathrm{P}_{1 L}$, middle loop $\mathrm{P}_{1 M}$, upper loop $\mathrm{P}_{1 U}$, and the prominence $\mathrm{P}_{2}$ are $\approx 220,260,340$, and $210 \mathrm{Mm}$, respectively. The magnetic field strength is calculated using the method proposed in Ofman \& Liu (2018) and Shen et al. (2019). The kink speed of the oscillations is defined as $C_{k}=2 L / P$, where $L$ and $P$ are the length of the magnetic field lines and period of the oscillation, respectively. The calculation gives the kink speed varying from $430-620 \mathrm{~km} \mathrm{~s}^{-1}$. The kink speed depends on the Alfvén speed as $C_{k}=V_{A} \sqrt{\frac{2}{1+n_{o} / n_{i}}}$, which is related to the magnetic field strength by the relation $V_{A}=\sqrt{\frac{B^{2}}{4 \pi \rho}}$. Here, $n_{o} / n_{i}$ and $\rho$ are the ratio of number density outside/inside the prominence and the mass density of the prominence, respectively. Assuming the ratio $n_{o} / n_{i}=0.01$, we find the Alfvén speed in the range of $307-441 \mathrm{~km} \mathrm{~s}^{-1}$. It is noted that even $n_{o} / n_{i}$ increases by 10 times, i.e., $n_{o} / n_{i}=0.1$, the derived Alfvén speed would increase by only $4 \%$. Therefore, the influence of the density ratio on the result is negligible. From the calculated Alfvén speed and mean mass density, $\rho=$ $1.67 \times 10^{-14} \mathrm{~g} \mathrm{~cm}^{-3}$ from Labrosse et al. (2010), the magnetic field strength in $\mathrm{P}_{1 L}, \mathrm{P}_{1 M}, \mathrm{P}_{1 U}$, and $\mathrm{P}_{2}$ is found to be $14.4 \pm 1.3$, $20.1 \pm 1.8,16.3 \pm 1.6$, and $14.4 \pm 1.2 \mathrm{G}$, respectively. All the calculated and derived parameters are listed in Table 1.

The prominence oscillation is due to stationary waves. In contrast, a traveling wave was also observed to propagate along the prominence layer $P_{1 U}$ with an average velocity of $\approx 435$ $\mathrm{km} \mathrm{s}^{-1}$ as indicated in the online animation of Fig. 3, which provides another method to estimate the magnetic field of the prominence as demonstrated by Shen et al. (2019).For this method, we assume that the average speed of the fast-mode EUV wave is of the order of Alfvén speed, i.e. $V_{\text {avg }} \sim V_{A}$ in the coronal conditions, where the sound speed is smaller than the Alfvén speed (see Shen et al., 2019). Using this assumption, we find that the magnetic field strength of the prominences is about $20 \mathrm{G}$, which is close to the magnetic field strength computed by first method. The difference in magnetic field measurement could be due to the uncertainties in the field-line lengths and the fast-mode wave speed measurements.

Our estimated magnetic field strengths are comparable with the previously reported values based on coronal seismology or prominence seismology (Vršnak et al., 2007; De Moortel \& Pascoe, 2009; Liu et al., 2012b; Xue et al., 2014; Pant et al., 2015; Zhang et al., 2017b; Luna et al., 2018; Mackay et al., 2020). Luna et al. (2018) did a statistical study using the GONG $\mathrm{H} \alpha$ observations of 196 solar filaments near the maximum of solar cycle 24 and they found that the minimum average magnetic field strength is about $16 \mathrm{G}$. Using the Hanle effect, the average field strength of prominences was measured to vary from 5 to 10 G (Leroy et al., 1983, 1984; Kuckein et al., 2009; Sasso et al., 2011; Kuckein et al., 2012; Xu et al., 2012; Raouafi et al., 2016). It seems that the observed magnetic field strength using Hanle effect is slightly less than derived by coronal seismology.

\section{Discussion and Summary}

The present analysis explores the oscillations of prominences situated above the East limb on 2011 February 11. These oscillations are initiated by an EUV wave associated with the eruption of another prominence. Since the oscillations are normal to the prominence layers therefore these are transverse oscillations. The main points of our study are as follows :

- Both the prominence with three layers near the source eruption region and another prominence further south oscillate in response to the fast-mode EUV wave associated with the eruption.

- For the $\mathrm{P}_{1 L}, \mathrm{P}_{1 M}$, and $\mathrm{P}_{1 U}$, the periods are 17,14 , and 22 minutes, respectively. As expected, for the three layers of the first oscillating prominence, the period is the longest for the uppermost layer.

- We have computed the coronal magnetic field strength of the prominences with two different methods with comparable results. The derived value of the magnetic field strength ranges from 15 to $21 \mathrm{G}$.

The oscillations in the solar atmosphere are commonly observed in different features like coronal loops, prominences, cavities, coronal streamers, etc. Mostly these oscillations are activated by external perturbations. EUV waves are one of the prominent causes for creating the oscillation in prominences 

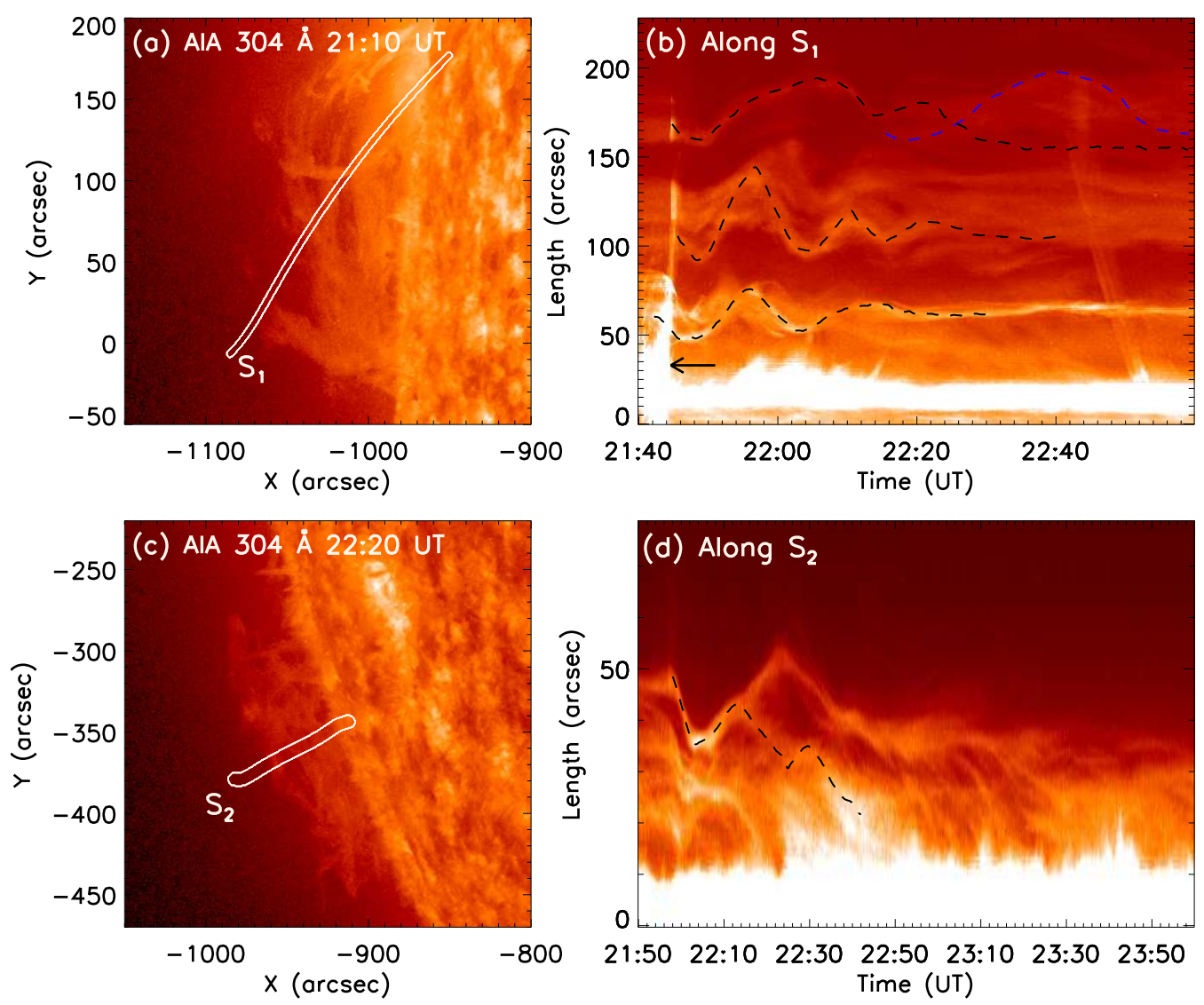

Fig. 3. Slices $S_{1}$ and $S_{2}$ along the directions of the oscillating prominences $P_{1}$ and $P_{2}$ are displayed in panels (a) and (c), respectively. Panels (b) and (d) show the time-distance diagrams along slices $S_{1}$ and $S_{2}$, respectively. A black arrow in panel (b) denotes the EUV wave crossing the prominence threads. The black dashed lines in panels (b) and (d) are the tracking of the prominence oscillations from the time-distance plots. The blue dashed line in panel (b) represents the peak, in which we did not find the complete oscillation. It could be another thread of the prominence different from the oscillating one traced by the black dashed line. Therefore, we are not considering this for our period analysis. An animation of the this figure is available online.

(Asai et al., 2012; Gosain, 2012), cavities (Zhang \& Ji, 2018), and coronal loops (Ballai, 2007; Guo et al., 2015). The present observations and analysis indicate that transverse oscillations in prominences are triggered by a fast-mode EUV wave at the east limb. Asai et al. (2012) found the oscillations of a prominence and a filament triggered by an EUV wave as a fast-mode MHD wave with a velocity of about $570-800 \mathrm{~km} \mathrm{~s}^{-1}$. Gosain (2012) analysed a flare associated transverse oscillations in a quiescent prominence. Liu et al. (2012a) and Zhang \& Ji (2018) reported on transverse oscillations in a cavity which are triggered by nearby EUV wave.

In the present case, we find the oscillations in the prominences $\mathrm{P}_{1 L}, \mathrm{P}_{1 M}, \mathrm{P}_{1 U}$, and $\mathrm{P}_{2}$ by a single EUV wave (speed $\approx$ $445 \pm 6 \mathrm{~km} \mathrm{~s}^{-1}$ ), which hits these prominences during its propagation. The calculated periods are in range of $14-22 \mathrm{~min}$. This range is consistent with Asai et al. (2012), Gosain (2012), Shen et al. (2014a), and Shen et al. (2014c). Further, the magnetic field strength of the prominences is derived by assuming the oscillations to be kink-mode oscillations. The value for the prominence $P_{1 U}$ is roughly consistent with the result derived by an independent method, i.e. a traveling wave.

It is noted that all three layers of the first prominence were oscillating collectively. However, the prominence seismology
Table 1. The measured and derived parameters of the prominences.

\begin{tabular}{|c|c|c|c|}
\hline Prominence & $\begin{array}{c}\text { Length } \\
(\mathrm{Mm})\end{array}$ & $\begin{array}{c}\text { Period } \\
(\mathrm{min})\end{array}$ & $\begin{array}{c}\text { Derived } \\
\text { magnetic field }(\mathrm{G})\end{array}$ \\
\hline $\mathrm{P}_{1 L}$ & 220 & $17 \pm 1.5$ & $14.4 \pm 1.3$ \\
$\mathrm{P}_{1 M}$ & 260 & $14 \pm 1.2$ & $20.1 \pm 1.8$ \\
$\mathrm{P}_{1 U}$ & 340 & $22 \pm 2.0$ & $16.3 \pm 1.6$ \\
$\mathrm{P}_{2}$ & 210 & $16 \pm 1.3$ & $14.4 \pm 1.2$ \\
\hline
\end{tabular}

in this paper is based on a single-segment configuration for the filament, which may introduce uncertainties in the estimate of the magnetic field. Considering that there exist thread-thread interactions (Zhou et al., 2017), it would be interesting to investigate their influence on the estimate of the prominence magnetic field in the future.

Acknowledgments: We thank both reviewers for their comments and suggestions. The authors thank the open data policy of the SDO team. PD thanks the CSIR, New Delhi for providing the Research Fellowship. PFC was supported by the National Key Research and Development Program of 

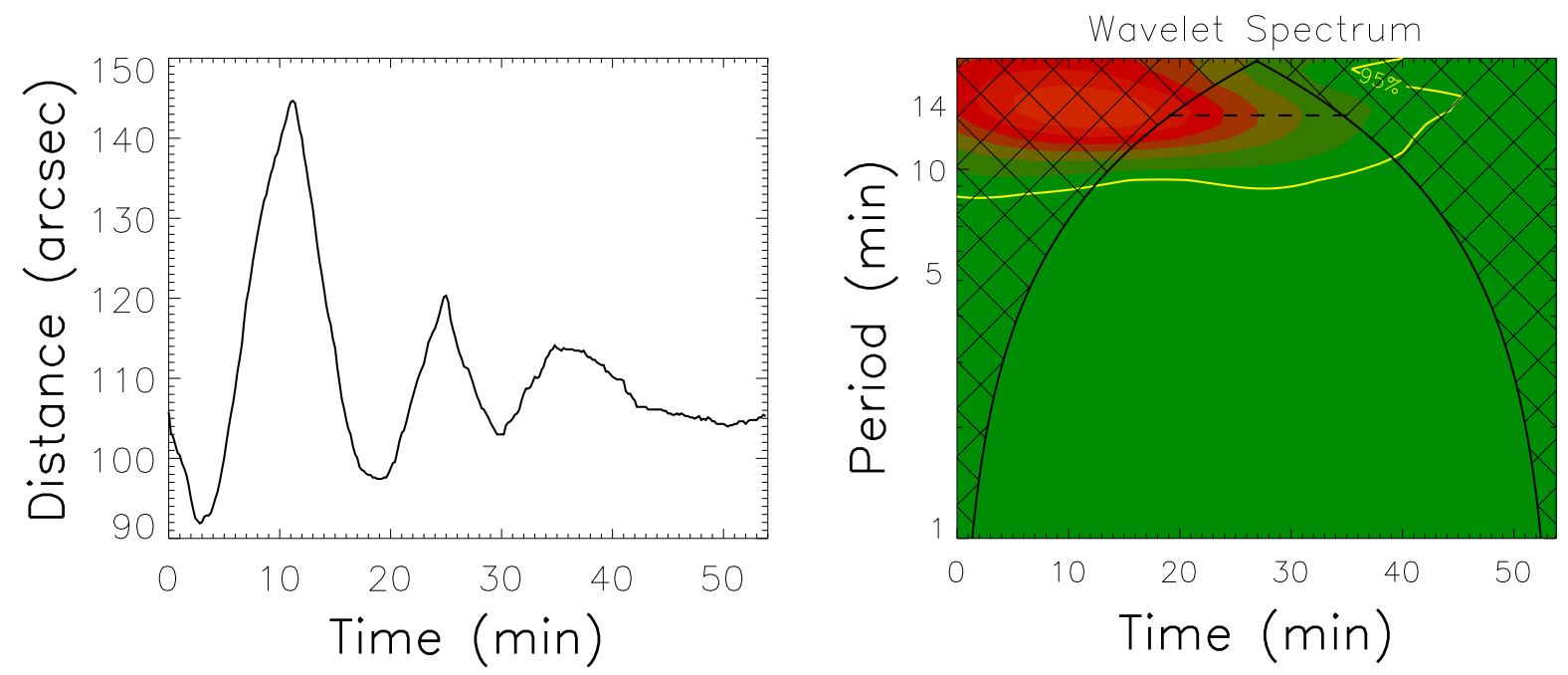

Fig. 4. Temporal evolution of the displacement of the prominence $\mathrm{P}_{1 M}$ (left panel). The right panel displays the wavelet spectrum, where the horizontal dashed line corresponds to the peak of global power spectrum at $14.0 \mathrm{~min}$. The starting time is 21:50 UT.

China (2020YFC2201200) and NSFC (11961131002), RC acknowledges the support from Bulgarian Science Fund under Indo-Bulgarian bilateral project, DST/INT/BLR/P-11/2019. YJM was supported by the Basic Science Research Program through the NRF funded by the Ministry of Education (NRF2019R1A2C1002634). We thank Dr. Manuel Luna for discussions on timeslice analysis.

\section{References}

Arregui, I., Oliver, R., \& Ballester, J. L. (2018). Prominence oscillations. Living Reviews in Solar Physics, 15(1), 3. doi:10.1007/s41116-018-0012-6.

Asai, A., Ishii, T. T., Isobe, H., Kitai, R., Ichimoto, K., UeNo, S., Nagata, S., Morita, S., Nishida, K., Shiota, D., Oi, A., Akioka, M., \& Shibata, K. (2012). First Simultaneous Observation of an $\mathrm{H} \alpha$ Moreton Wave, EUV Wave, and Filament/Prominence Oscillations. Astrophys. J. Lett., 745(2), L18. doi:10. 1088/2041-8205/745/2/L18. arXiv:1112.5915.

Aulanier, G., DeLuca, E. E., Antiochos, S. K., McMullen, R. A., \& Golub, L. (2000). The Topology and Evolution of the Bastille Day Flare. Astrophys. J., 540(2), 1126-1142. doi:10.1086/309376.

Ballai, I. (2007). Global Coronal Seismology. Solar Phys., 246(1), 177-185. doi:10.1007/s11207-007-0415-3. arXiv:0704.1398.

Chandra, R., Chen, P. F., Devi, P., Joshi, R., Schmieder, B., Moon, Y.J., \& Uddin, W. (2021a). Fine Structures of an EUV Wave Event from Multi-viewpoint Observations. Astrophys. J., 919(1), 9. doi:10.3847/ 1538-4357/ac1077. arXiv:2106.14024.

Chandra, R., Chen, P. F., Fulara, A., Srivastava, A. K., \& Uddin, W. (2016). Peculiar Stationary EUV Wave Fronts in the Eruption on 2011 May 11. Astrophys. J., 822(2), 106. doi:10.3847/0004-637X/822/2/106. arXiv: 1602.08693.

Chandra, R., Démoulin, P., Devi, P., Joshi, R., \& Schmieder, B. (2021b). Filament Eruption Driving EUV Loop Contraction then Expansion above a Stable Filament. arXiv e-prints, (p. arXiv:2109.07821). arXiv : 2109.07821.

Chen, P. F., Fang, C., Chandra, R., \& Srivastava, A. K. (2016). Can a Fast-Mode EUV Wave Generate a Stationary Front? Solar Phys., 291(11), 3195-3206. doi:10.1007/s11207-016-0920-3. arXiv: 1604.07982.

Chen, P. F., Fang, C., \& Shibata, K. (2005). A Full View of EIT Waves. Astrophys. J., 622(2), 1202-1210. doi:10.1086/428084.

Chen, P. F., Wu, S. T., Shibata, K., \& Fang, C. (2002). Evidence of EIT and Moreton Waves in Numerical Simulations. Astrophys. J. Lett., 572, L99L102. doi:10.1086/341486.
Chen, P. F., \& Wu, Y. (2011). First Evidence of Coexisting EIT Wave and Coronal Moreton Wave from SDO/AIA Observations. Astrophys. J. Lett., 732(2), L20. doi:10.1088/2041-8205/732/2/L20. arXiv:1103. 0871.

Chen, P.-F., Xu, A.-A., \& Ding, M.-D. (2020). Some interesting topics provoked by the solar filament research in the past decade. Research in Astronomy and Astrophysics, 20(10), 166. doi:10.1088/1674-4527/20/10/ 166. arXiv: 2010.02462.

Cheng, X., Zhang, J., Kliem, B., Török, T., Xing, C., Zhou, Z. J., Inhester, B., \& Ding, M. D. (2020). Initiation and Early Kinematic Evolution of Solar Eruptions. Astrophys. J., 894(2), 85. doi:10.3847/1538-4357/ab886a. arXiv: 2004.03790.

De Moortel, I., \& Pascoe, D. J. (2009). Putting Coronal Seismology Estimates of the Magnetic Field Strength to the Test. Astrophys. J. Lett., 699(2), L72L75. doi:10.1088/0004-637X/699/2/L72.

Delannée, C. (2000). Another View of the EIT Wave Phenomenon. Astrophys. J., 545(1), 512-523. doi:10.1086/317777.

Delannée, C., \& Aulanier, G. (1999). Cme Associated with Transequatorial Loops and a Bald Patch Flare. Solar Phys., 190, 107-129. doi:10.1023/A : 1005249416605.

Gosain, S. (2012). Evidence for Collapsing Fields in the Corona and Photosphere during the 2011 February 15 X2.2 Flare: SDO/AIA and HMI Observations. Astrophys. J., 749(1), 85. doi:10.1088/0004-637X/749/1/85. arXiv: 1202.1784.

Guo, Y., Erdélyi, R., Srivastava, A. K., Hao, Q., Cheng, X., Chen, P. F., Ding, M. D., \& Dwivedi, B. N. (2015). Magnetohydrodynamic Seismology of a Coronal Loop System by the First Two Modes of Standing Kink Waves. Astrophys. J., 799(2), 151. doi:10.1088/0004-637X/799/2/151. arXiv: 1411.7095.

Hyder, C. L. (1966). Winking Filaments and Prominence and Coronal Magnetic Fields. Zeitschrift für Astrophysik, 63, 78-84.

Joshi, B., Kushwaha, U., Veronig, A. M., Dhara, S. K., Shanmugaraju, A., \& Moon, Y.-J. (2017). Formation and Eruption of a Flux Rope from the Sigmoid Active Region NOAA 11719 and Associated M6.5 Flare: A Multiwavelength Study. Astrophys. J., 834(1), 42. doi:10.3847/1538-4357/ 834/1/42. arXiv: 1701.00967.

Joshi, N. C., Srivastava, A. K., Filippov, B., Uddin, W., Kayshap, P., \& Chandra, R. (2013). A Study of a Failed Coronal Mass Ejection Core Associated with an Asymmetric Filament Eruption. Astrophys. J., 771(1), 65. doi:10.1088/0004-637X/771/1/65. arXiv: 1304.6852.

Joshi, R., Chandra, R., Schmieder, B., Moreno-Insertis, F., Aulanier, G., Nóbrega-Siverio, D., \& Devi, P. (2020). Case study of multi-temperature coronal jets for emerging flux MHD models. Astron. Astrophys., 639, A22. doi:10.1051/0004-6361/202037806. arXiv: 2005.06064.

Kleczek, J., \& Kuperus, M. (1969). Oscillatory Phenomena in Quiescent Prominences. Solar Phys., 6(1), 72-79. doi:10.1007/BF00146797. 
Kuckein, C., Centeno, R., Martínez Pillet, V., Casini, R., Manso Sainz, R., \& Shimizu, T. (2009). Magnetic field strength of active region filaments. Astron. Astrophys., 501(3), 1113-1121. doi:10.1051/0004-6361/ 200911800. arXiv:0904. 4876.

Kuckein, C., Martínez Pillet, V., \& Centeno, R. (2012). An active region filament studied simultaneously in the chromosphere and photosphere. I. Magnetic structure. Astron. Astrophys., 539, A131. doi:10.1051/0004-6361/ 201117675. arXiv:1112.1672.

Labrosse, N., Heinzel, P., Vial, J. C., Kucera, T., Parenti, S., Gunár, S., Schmieder, B., \& Kilper, G. (2010). Physics of Solar Prominences: I-Spectral Diagnostics and Non-LTE Modelling. Space Sci. Rev., 151(4), 243-332. doi:10.1007/s11214-010-9630-6. arXiv:1001.1620.

Lemen, J. R., Title, A. M., Akin, D. J., Boerner, P. F., Chou, C., Drake, J. F., Duncan, D. W., Edwards, C. G., Friedlaender, F. M., Heyman, G. F., Hurlburt, N. E., Katz, N. L., Kushner, G. D., Levay, M., Lindgren, R. W., Mathur, D. P., McFeaters, E. L., Mitchell, S., Rehse, R. A., Schrijver, C. J., Springer, L. A., Stern, R. A., Tarbell, T. D., Wuelser, J.-P., Wolfson, C. J., Yanari, C., Bookbinder, J. A., Cheimets, P. N., Caldwell, D., Deluca, E. E., Gates, R., Golub, L., Park, S., Podgorski, W. A., Bush, R. I., Scherrer, P. H., Gummin, M. A., Smith, P., Auker, G., Jerram, P., Pool, P., Soufli, R., Windt, D. L., Beardsley, S., Clapp, M., Lang, J., \& Waltham, N. (2012). The Atmospheric Imaging Assembly (AIA) on the Solar Dynamics Observatory (SDO). Solar Phys., 275, 17-40. doi:10.1007/s11207-011-9776-8.

Leroy, J. L., Bommier, V., \& Sahal-Brechot, S. (1983). The Magnetic Field in the Prominences of the Polar Crown. Solar Phys., 83(1), 135-142. doi:10. $1007 / \mathrm{BF} 00148248$

Leroy, J. L., Bommier, V., \& Sahal-Brechot, S. (1984). New data on the magnetic structure of quiescent prominences. Astron. Astrophys., 131(1), 33-44.

Liakh, V., Luna, M., \& Khomenko, E. (2020). Numerical simulations of largeamplitude oscillations in flux rope solar prominences. Astron. Astrophys. 637, A75. doi:10.1051/0004-6361/201937083. arXiv : 2003.04343.

Liu, R., Liu, C., Török, T., Wang, Y., \& Wang, H. (2012a). Contracting and Erupting Components of Sigmoidal Active Regions. Astrophys. J., 757(2), 150. doi:10.1088/0004-637X/757/2/150. arXiv: 1208.0640 .

Liu, R., Wang, H., \& Alexander, D. (2009). Implosion in a Coronal Eruption. Astrophys. J., 696(1), 121-135. doi:10.1088/0004-637X/696/1/121.

Liu, W., Ofman, L., Nitta, N. V., Aschwanden, M. J., Schrijver, C. J., Title, A. M., \& Tarbell, T. D. (2012b). Quasi-periodic Fast-mode Wave Trains within a Global EUV Wave and Sequential Transverse Oscillations Detected by SDO/AIA. Astrophys. J., 753(1), 52. doi:10.1088/0004-637X/753/ 1/52. arXiv: 1204.5470.

Luna, M. (2016). Large-Amplitude Oscillations in Prominences. In W. D. Pesnell, \& B. Thompson (Eds.), SDO 2016: Unraveling the Sun's Complexity (p. 60). Proceedings of the conference held 17-21 October, 2016 in Burlington, VT. Online at http://sdo-2016.1ws-sdo-workshops.org/.

Luna, M., \& Karpen, J. (2012). Large-amplitude Longitudinal Oscillations in a Solar Filament. Astrophys. J. Lett., 750(1), L1. doi:10.1088/2041-8205/ 750/1/L1. arXiv: 1203.5027.

Luna, M., Karpen, J., Ballester, J. L., Muglach, K., Terradas, J., Kucera, T., \& Gilbert, H. (2018). GONG Catalog of Solar Filament Oscillations Near Solar Maximum. Astrophys. J. Suppl, 236(2), 35. doi:10.3847/1538-4365/ aabde7. arXiv: 1804.03743

Luna, M., Karpen, J., Díaz, A., Knizhnik, K., Muglach, K., Gilbert, H., \& Kucera, T. (2014). Large-amplitude longitudinal oscillations in solar prominences. In B. Schmieder, J.-M. Malherbe, \& S. T. Wu (Eds.), Nature of Prominences and their Role in Space Weather (pp. 155-158). Proceedings of the International Astronomical Union, IAU Symposium, Cambridge University Press, volume 8. doi:10.1017/S1743921313010909.

Luna, M., \& Moreno-Insertis, F. (2021). Large-amplitude Prominence Oscillations following Impact by a Coronal Jet. Astrophys. J., 912(1), 75 doi:10.3847/1538-4357/abec46. arXiv: 2103.02661.

Luna, M., Su, Y., Schmieder, B., Chandra, R., \& Kucera, T. A. (2017). Largeamplitude Longitudinal Oscillations Triggered by the Merging of Two Solar Filaments: Observations and Magnetic Field Analysis. Astrophys. J., 850(2), 143. doi:10.3847/1538-4357/aa9713. arXiv: 1711.01038.

Mackay, D. H., Schmieder, B., López Ariste, A., \& Su, Y. (2020). Modelling and observations: Comparison of the magnetic field properties in a prominence. Astron. Astrophys., 637, A3. doi:10.1051/0004-6361/ 201936656.

Mazumder, R., Pant, V., Luna, M., \& Banerjee, D. (2020). Simultaneous longitudinal and transverse oscillations in filament threads after a failed eruption. Astron. Astrophys., 633, A12. doi:10.1051/0004-6361/ 201936453. arXiv: 1910.11260

Nakariakov, V. M., Anfinogentov, S. A., Antolin, P., Jain, R., Kolotkov, D. Y., Kupriyanova, E. G., Li, D., Magyar, N., Nisticò, G., Pascoe, D. J., Srivastava, A. K., Terradas, J., Vasheghani Farahani, S., Verth, G., Yuan, D., \& Zimovets, I. V. (2021). Kink Oscillations of Coronal Loops. Space Sci. Rev., 217(6), 73. doi:10.1007/s11214-021-00847-2. arXiv:2109.11220.

Nakariakov, V. M., \& Verwichte, E. (2005). Coronal Waves and Oscillations. Living Reviews in Solar Physics, 2(1), 3. doi:10.12942/lrsp-2005-3.

Ofman, L., \& Liu, W. (2018). Quasi-periodic Counter-propagating Fast Magnetosonic Wave Trains from Neighboring Flares: SDO/AIA Observations and 3D MHD Modeling. Astrophys. J., 860(1), 54. doi:10.3847/1538-4357/ aac2e8. arXiv: 1805.00365.

Ofman, L., \& Thompson, B. J. (2002). Interaction of EIT Waves with Coronal Active Regions. Astrophys. J., 574(1), 440-452. doi:10.1086/340924.

Okamoto, T. J., Nakai, H., Keiyama, A., Narukage, N., UeNo, S., Kitai, R., Kurokawa, H., \& Shibata, K. (2004). Filament Oscillations and Moreton Waves Associated with EIT Waves. Astrophys. J., 608(2), 1124-1132. doi:10.1086/420838.

Pant, V., Mazumder, R., Yuan, D., Banerjee, D., Srivastava, A. K., \& Shen, Y. (2016). Simultaneous Longitudinal and Transverse Oscillations in an Active-Region Filament. Solar Phys., 291(11), 3303-3315. doi:10.1007/ s11207-016-1018-7. arXiv: 1611.03984.

Pant, V., Srivastava, A. K., Banerjee, D., Goossens, M., Chen, P.-F., Joshi, N. C., \& Zhou, Y.-H. (2015). MHD Seismology of a loop-like filament tube by observed kink waves. Research in Astronomy and Astrophysics, 15(10), 1713. doi:10.1088/1674-4527/15/10/008. arXiv: 1503.02281.

Pesnell, W. D., Thompson, B. J., \& Chamberlin, P. C. (2012). The Solar Dynamics Observatory (SDO). Solar Phys., 275, 3-15. doi:10.1007/ s11207-011-9841-3.

Raouafi, N. E., Riley, P., Gibson, S., Fineschi, S., \& Solanki, S. K. (2016). Diagnostics of Coronal Magnetic Fields Through the Hanle Effect in UV and IR Lines. Frontiers in Astronomy and Space Sciences, 3, 20. doi:10. 3389/fspas. 2016.00020. arXiv: 1606.08493.

Roudier, T., Schmieder, B., Filippov, B., Chandra, R., \& Malherbe, J. M. (2018). Horizontal photospheric flows trigger a filament eruption. Astron. Astrophys., 618, A43. doi:10.1051/0004-6361/201832937. arXiv: 1808.02272

Sasso, C., Lagg, A., \& Solanki, S. K. (2011). Multicomponent He I $10830 \AA$ profiles in an active filament. Astron. Astrophys., 526, A42. doi:10.1051/ 0004-6361/200912956. arXiv: 1101.5563.

Schmieder, B., Aulanier, G., \& Vršnak, B. (2015). Flare-CME Models: An Observational Perspective (Invited Review). Solar Phys., 290(12), 34573486. doi:10.1007/s11207-015-0712-1.

Shen, Y., Chen, P. F., Liu, Y. D., Shibata, K., Tang, Z., \& Liu, Y. (2019). First Unambiguous Imaging of Large-scale Quasi-periodic Extreme-ultraviolet Wave or Shock. Astrophys. J., 873(1), 22. doi:10.3847/1538-4357/ ab01dd. arXiv: 1901.08199.

Shen, Y., Ichimoto, K., Ishii, T. T., Tian, Z., Zhao, R., \& Shibata, K. (2014a). A Chain of Winking (Oscillating) Filaments Triggered by an Invisible Extreme-ultraviolet Wave. Astrophys. J., 786(2), 151. doi:10.1088/ 0004-637X/786/2/151. arXiv: 1403.7705.

Shen, Y., Liu, Y. D., Chen, P. F., \& Ichimoto, K. (2014b). Simultaneous Transverse Oscillations of a Prominence and a Filament and Longitudinal Oscillation of Another Filament Induced by a Single Shock Wave. Astrophys. J., 795(2), 130. doi:10.1088/0004-637X/795/2/130. arXiv: 1409.1304.

Shen, Y., Liu, Y. D., Chen, P. F., \& Ichimoto, K. (2014c). Simultaneous Transverse Oscillations of a Prominence and a Filament and Longitudinal Oscillation of Another Filament Induced by a Single Shock Wave. Astrophys. J., 795(2), 130. doi:10.1088/0004-637X/795/2/130. arXiv:1409.1304.

Srivastava, A. K., Singh, T., Ofman, L., \& Dwivedi, B. N. (2016). Inference of magnetic field in the coronal streamer invoking kink wave motions generated by multiple EUV waves. Mon. Not. Roy. Astron. Soc., 463(2), 1409-1415. doi:10.1093/mnras/stw2017. arXiv:1606.00337.

Thompson, B. J., Plunkett, S. P., Gurman, J. B., Newmark, J. S., St. Cyr, O. C., \& Michels, D. J. (1998). SOHO/EIT observations of an Earth-directed coronal mass ejection on May 12, 1997. Geophys. Res. Lett., 25(14), 2465-2468. doi:10.1029/98GL50429.

Thompson, W. T., \& Schmieder, B. (1991). Oscillations in $\mathrm{H} \alpha$ filaments: center-to-limb study. Astron. Astrophys., 243(2), 501-511.

Torrence, C., \& Compo, G. P. (1998). A Practical Guide to Wavelet Analysis. 
Bulletin of the American Meteorological Society, 79, 61-78. doi:10.1175/ 1520-0477 (1998) 079<0061: APGTWA>2 .0.CO;2.

Vršnak, B. (1993). Classification of Prominence Oscillations. Hvar Observatory Bulletin, 17(1), 23.

Vršnak, B., Veronig, A. M., Thalmann, J. K., \& Žic, T. (2007). Large amplitude oscillatory motion along a solar filament. Astron. Astrophys., 471(1), 295299. doi:10.1051/0004-6361:20077668. arXiv:0707.1752.

Vršnak, B., Warmuth, A., Brajša, R., \& Hanslmeier, A. (2002). Flare waves observed in Helium I $10830 \AA$. A link between $\mathrm{H} \alpha$ Moreton and EIT waves. Astron. Astrophys., 394, 299-310. doi:10.1051/0004-6361:20021121.

Wang, Y. M. (2000). EIT Waves and Fast-Mode Propagation in the Solar Corona. Astrophys. J. Lett., 543(1), L89-L93. doi:10.1086/318178.

Wu, S. T., Zheng, H., Wang, S., Thompson, B. J., Plunkett, S. P., Zhao, X. P., \& Dryer, M. (2001). Three-dimensional numerical simulation of MHD waves observed by the Extreme Ultraviolet Imaging Telescope. J. Geophys. Res., 106(A11), 25089-25102. doi:10.1029/2000JA000447.

Xu, Z., Lagg, A., Solanki, S., \& Liu, Y. (2012). Magnetic Fields of an Active Region Filament from Full Stokes Analysis of Si I $1082.7 \mathrm{~nm}$ and He I $1083.0 \mathrm{~nm}$. Astrophys. J., 749(2), 138. doi:10.1088/0004-637X/749/2/ 138. arXiv: 1202.4562.

Xue, Z. K., Yan, X. L., Qu, Z. Q., \& Zhao, L. (2014). Transverse Oscillation of a Filament Triggered by an Extreme Ultraviolet Wave. In K. N. Nagendra, J. O. Stenflo, Z. Q. Qu, \& M. Sampoorna (Eds.), Solar Polarization 7 (p. 53). ASP, San Francisco, volume 489 of Astronomical Society of the Pacific Conference Series.

Zhang, L. Y., Fang, C., \& Chen, P. F. (2019). Damping Mechanisms of the Solar Filament Longitudinal Oscillations in the Weak Magnetic Field. Astrophys. J., 884(1), 74. doi:10.3847/1538-4357/ab3d3a. arXiv: 1908.07148.

Zhang, Q. M., Chen, P. F., Xia, C., \& Keppens, R. (2012). Observations and simulations of longitudinal oscillations of an active region prominence. Astron. Astrophys., 542, A52. doi:10.1051/0004-6361/ 201218786. arXiv: 1204.3787.

Zhang, Q. M., Guo, J. H., Tam, K. V., \& Xu, A. A. (2020). Longitudinal filament oscillations enhanced by two C-class flares. Astron. Astrophys., 635, A132. doi:10.1051/0004-6361/201937291. arXiv: 2001.01250.

Zhang, Q. M., \& Ji, H. S. (2018). Vertical Oscillation of a Coronal Cavity Triggered by an EUV Wave. Astrophys. J., 860(2), 113. doi:10.3847/ 1538-4357/aac37e. arXiv: 1805.01088.

Zhang, Q. M., Li, D., \& Ning, Z. J. (2017a). Simultaneous Transverse and Longitudinal Oscillations in a Quiescent Prominence Triggered by a Coronal Jet. Astrophys. J., 851(1), 47. doi:10.3847/1538-4357/aa9898. arXiv: 1711.00670 .

Zhang, Q. M., Li, D., \& Ning, Z. J. (2017b). Simultaneous Transverse and Longitudinal Oscillations in a Quiescent Prominence Triggered by a Coronal Jet. Astrophys. J., 851(1), 47. doi:10.3847/1538-4357/aa9898. arXiv: 1711.00670 .

Zhang, Y., \& Engvold, O. (1991). Vertical velocities and oscillations in quiescent filaments. Solar Phys., 134(2), 275-286. doi:10.1007/BF00152648.

Zhao, X. H., Wu, S. T., Wang, A. H., Vourlidas, A., Feng, X. S., \& Jiang, C. W. (2011). Uncovering the Wave Nature of the EIT Wave for the 2010 January 17 Event through Its Correlation to the Background Magnetosonic Speed. Astrophys. J., 742(2), 131. doi:10.1088/0004-637X/742/2/131.

Zheng, R., Chen, Y., Wang, B., \& Song, H. (2020). An Extreme Ultraviolet Wave Associated with a Solar Filament Activation. Astrophys. J., 894(2), 139. doi:10.3847/1538-4357/ab863c. arXiv: 2004.04904.

Zheng, R., Jiang, Y., Yang, J., Bi, Y., Hong, J., Yang, D., \& Yang, B. (2012). A Fast Propagating Extreme-Ultraviolet Wave Associated with a Mini-filament Eruption. Astrophys. J., 753(2), 112. doi:10.1088/0004-637X/753/2/ 112.

Zhou, G. P., Zhang, J., \& Wang, J. X. (2016). OBSERVATIONS OF MAGNETIC FLUX-ROPE OSCILLATION DURING THE PRECURSOR PHASE OF a SOLAR ERUPTION. The Astrophysical Journal, 823(1), L19. URL: https://doi.org/10.3847/2041-8205/823/1/ 119. doi:10.3847/2041-8205/823/1/119.

Zhou, Y.-H., Xia, C., Keppens, R., Fang, C., \& Chen, P. F. (2018). Threedimensional MHD Simulations of Solar Prominence Oscillations in a Magnetic Flux Rope. Astrophys. J., 856(2), 179. doi:10.3847/1538-4357/ aab614. arXiv: 1803.03385.

Zhou, Y.-H., Zhang, L.-Y., Ouyang, Y., Chen, P. F., \& Fang, C. (2017). Solar Filament Longitudinal Oscillations along a Magnetic Field Tube with Two Dips. Astrophys. J., 839(1), 9. doi:10.3847/1538-4357/aa67de.
arXiv: 1703.06560.

Zhukov, A. N., \& Auchère, F. (2004). On the nature of EIT waves, EUV dimmings and their link to CMEs. Astron. Astrophys., 427, 705-716. doi:10.1051/0004-6361:20040351. 